\title{
Optimal Transport between Gaussian Stationary Processes
}

\author{
Mattia Zorzi
}

\begin{abstract}
We consider the optimal transport problem between multivariate Gaussian stationary stochastic processes. The transportation effort is the variance of the filtered discrepancy process. The main contribution of this technical note is to show that the corresponding solution leads to a weighted Hellinger distance between multivariate power spectral densities. Then, we propose a spectral estimation approach in the case of indirect measurements which is based on this distance.
\end{abstract}

Index Terms-Optimal transport, convex optimization, generalized covariance extension problem, spectral analysis.

\section{INTRODUCTION}

The optimal mass transport problem recently gained attention in the control filed. Its modern formulation, due to Kantorovich [12], consists in minimizing the effort of transporting one nonnegative measure to another nonnegative given an associated cost of moving mass from a point to another one. The latter has been used to derive new distances between covariance matrices and spectral densities [10], [4]. Matrix-valued transport problems have been proposed in [15], [3] which lead to transportation distances between multivariate power spectral densities. The key idea in these works is to set up the optimal transport problem in terms of the joint power spectral density. It is worth noting that the latter gives a complete joint description of two zero-mean multivariate Gaussian stationary stochastic processes.

In the present paper, instead, we face the optimal transport problem in a different way. More precisely, such a problem is formulated directly in terms of the joint probability density of two Gaussian stationary processes. The transportation cost is the variance of the filtered discrepancy process, i.e. the difference between the two processes. The main contribution of this technical note is to show that the corresponding solution leads to a weighted Hellinger distance between multivariate power spectral densities. The latter has been introduced in [6], [17] for the case without weight function. It is worth noticing that the aforementioned correspondence can be regarded as the dynamic extension of to the results showed in [16], [14] for zeromean Gaussian random vectors.

Then, we show that the weighted transportation distance can be used to perform spectral estimation in the case we have indirect measurements. More precisely, we have a network of $m$ sensors which measure indirectly $m$ variables of interest for which we want to estimate the spectral density. The matrix-valued weight function of our distance is given by the transfer matrix characteristic of the sensor network. We consider the THREE-like spectral estimation paradigm for which a large body of literature for the scalar [1], [11], [2], [13], [24], multivariate [5], [26], [23], [20], [21] and multidimensional case [9], [18], [19], [22] has been developed. Here, the estimated spectral density is the closest one to a prior spectral density and matching the output covariance of a bank of filters (fed with the measured data). The prior represents the priori information that we have about the spectrum. The bank of filter is designed by the user in such a way the estimator exhibit high-resolution properties in prescribed frequency

M. Zorzi is with the Dipartimento di Ingegneria dell'Informazione, Università degli studi di Padova, via Gradenigo 6/B, 35131 Padova, Italy (zorzimat@dei.unipd.it). This work was partially supported by the SID project "A Multidimensional and Multivariate Moment Problem Theory for Target Parameter Estimation in Automotive Radars" (ZORZ SID19 01) funded by the Department of Information Engineering of the University of Padova. bands, [1]. Finally, we compare the proposed estimator with the one obtained by using the Itakura-Saito distance. The latter has been in introduced in [5].

The outline of the technical note is as follows. In Section $\amalg$ we introduce the optimal transport problem between Gaussian processes and we derive the corresponding weighted Hellinger distance. Section III is devoted to spectral estimation with indirect measurements. In Section IV we provide a simulation study to test the proposed estimator. Finally, in Section $\mathrm{V}$ we draw the conclusions.

The following notation will be adopted throughout the paper. $\mathcal{Q}_{n}$ denotes the space of symmetric matrices of dimension $n \times n$. Given an Hermitian matrix $X, X>0(X \geq 0)$ means that $X$ is positive (semi-)definite. Given $X \geq 0, X^{1 / 2}$ denotes a square root of $X$, i.e. $X=X^{1 / 2} X^{1 / 2}$. Given a matrix $X \in \mathbb{C}^{n \times n}:[X]_{i l}$ denotes its entry in position $(i, l)$; we define the norms $\|X\|:=\sqrt{\operatorname{tr}\left(X^{*} X\right)}$ and $\|X\|_{W}:=\sqrt{\operatorname{tr}\left(X^{*} W X\right)}$ with $W=W^{*}>0$. We define the unit circle as $\mathbb{T}=\left\{e^{j \vartheta}\right.$ s.t. $\left.\vartheta \in[0,2 \pi)\right\} . \mathbb{L}_{\infty}^{m \times m}(\mathbb{T})$ denotes the function space of matrix functions defined on $\mathbb{T}$ and taking values in $\mathbb{C}^{n \times n}$. Given $\Phi, \Psi \in \mathbb{L}_{\infty}^{m \times m}(\mathbb{T})$ : the shorthand notation $\int_{\mathbb{T}} \Phi$ denotes the integration of $\Phi$ on $\mathbb{T}$ with respect to the normalized Lebesgue measure; $\Phi>0$ means that $\Phi\left(e^{j \vartheta}\right)>0$ for any $e^{j \vartheta} \in \mathbb{T}$ almost everywhere, $\Phi=\Psi$ means that $\Phi$ and $\Psi$ coincide almost everywhere. $\mathcal{S}_{m}^{+}$denotes the set of spectral densities bounded and coercive of dimension $m \times m$. Given a sequence $h=\left\{h_{t}, t \in \mathbb{Z}\right\}$, $(h)_{t}$ denotes $h_{t}$. Let $g$ and $h$ denote two sequences, then $g \star h$ denotes the discrete convolution operation.

\section{Transportation distance BetweEn GaUssian PROCESSES}

We consider two $m$-dimensional jointly Gaussian stationary stochastic processes $\mathbf{x}=\left\{\mathbf{x}_{t}, t \in \mathbb{Z}\right\}$ and $\mathbf{y}=\left\{\mathbf{y}_{t}, t \in \mathbb{Z}\right\}$ whose mean is equal to zero. The latter are completely described by the finite dimensional probability density functions $p_{\mathbf{x}}\left(x_{t}, x_{s} ; t, s\right)$ and $p_{\mathbf{y}}\left(y_{t}, y_{s} ; t, s\right)$ with $t, s \in \mathbb{Z}$. Let $p_{\mathbf{x}, \mathbf{y}}\left(x_{t}, x_{s}, y_{u}, y_{v} ; t, s, u, v\right)$, with $t, s, u, v \in \mathbb{Z}$, be the finite dimensional joint probability density of $\mathbf{x}$ and $\mathbf{y}$. Then, we consider the following optimal transport problem:

$$
d\left(p_{\mathbf{x}}, p_{\mathbf{y}}\right)^{2}=\inf _{p_{\mathbf{x}, \mathbf{y}} \in \mathcal{P}}\left\{\mathbb{E}\left[\left\|(h \star(\mathbf{x}-\mathbf{y}))_{t}\right\|^{2}\right] \text { s.t. 2)-3 hold }\right\}
$$

where $\mathcal{P}$ denotes the set of Gaussian joint probability densities $p_{\mathbf{x}, \mathbf{y}}$; $h=\left\{h_{t}, t \in \mathbb{Z}\right\}$, with $h_{t} \in \mathbb{R}^{m \times m}$ and $h_{t}=0$ for any $t<0$, represents the impulse of a $\mathrm{BIBO}$ system;

$$
\int_{\mathbb{R}^{m}} \int_{\mathbb{R}^{m}} p_{\mathbf{x}, \mathbf{y}}\left(x_{t}, x_{s}, y_{u}, y_{v} ; t, s, u, v\right) \mathrm{d} y_{u} \mathrm{~d} y_{v}=p_{\mathbf{x}}\left(x_{t}, x_{s} ; t, s\right),
$$

$\int_{\mathbb{R}^{m}} \int_{\mathbb{R}^{m}} p_{\mathbf{x}, \mathbf{y}}\left(x_{t}, x_{s}, y_{u}, y_{v} ; t, s, u, v\right) \mathrm{d} x_{t} \mathrm{~d} x_{s}=p_{\mathbf{y}}\left(y_{u}, y_{v} ; u, v\right)$,

for any $t, s, u, v \in \mathbb{Z}$. It is worth noting that (1) represents the optimal transport problem between Gaussian processes $\mathbf{x}$ and $\mathbf{y}$. The transportation cost is the variance of the filtered process $h \star(\mathbf{x}-\mathbf{y})$ and $\mathbf{x}-\mathbf{y}$ can be regarded as the discrepancy process. 
Example. In the case that $h$ is such that $h_{0}=I$ and $h_{t}=0$ for any $t \neq 0$, the transportation cost is the variance of the discrepancy process $\mathbf{x}-\mathbf{y}$. Then, we have

$$
d\left(p_{\mathbf{x}}, p_{\mathbf{y}}\right)=\left[\inf _{p_{\mathbf{x}, \mathbf{y}} \in \mathcal{P}}\left\{\mathbb{E}\left[\left\|\mathbf{x}_{t}-\mathbf{y}_{t}\right\|^{2}\right] \text { s.t. }[2]-(3) \text { hold }\right\}\right]^{1 / 2} .
$$

The latter is the dynamic counterpart of the 2-Wasserstein distance in [16] between Gaussian random vectors.

Let $\Phi_{\mathbf{x}}$ and $\Phi_{\mathbf{y}}$ denote the power spectral density of $\mathbf{x}$ and $\mathbf{y}$, respectively. The next result shows that the solution of the optimal transport problem in 11) can be expressed in terms of $\Phi_{\mathbf{x}}$ and $\Phi_{\mathbf{y}}$.

Proposition 2.1: Consider the optimization problem in (1). It holds that:

$$
d_{\Omega}\left(p_{\mathbf{x}}, p_{\mathbf{y}}\right)^{2}=\operatorname{tr} \int_{\mathbb{T}} \Omega \Phi_{\mathbf{x}}+\Omega \Phi_{\mathbf{y}}-2\left(\Phi_{\mathbf{y}}^{1 / 2} \Omega \Phi_{\mathbf{x}} \Omega \Phi_{\mathbf{y}}^{1 / 2}\right)^{1 / 2} .
$$

Proof: Consider a joint process $\left[\mathbf{x}^{T} \mathbf{y}^{T}\right]^{T}$ whose probability density belongs to $\mathcal{P}$ and satisfying (2)-3. Since the latter is Gaussian, an equivalent description for such a process is given by its power spectral density

$$
\Phi=\left[\begin{array}{cc}
\Phi_{\mathbf{x}} & \Phi_{\mathbf{x y}} \\
\Phi_{\mathbf{y x}} & \Phi_{\mathbf{y}}
\end{array}\right]
$$

Notice that

$$
\begin{aligned}
& \mathbb{E}\left[(h \star \mathbf{x})_{t}(h \star \mathbf{x})_{t}^{T}\right]=\int_{\mathbb{T}} H \Phi_{\mathbf{x}} H^{*} \\
& \mathbb{E}\left[(h \star \mathbf{y})_{t}(h \star \mathbf{y})_{t}^{T}\right]=\int_{\mathbb{T}} H \Phi_{\mathbf{y}} H^{*} \\
& \mathbb{E}\left[(h \star \mathbf{x})_{t}(h \star \mathbf{y})_{t}^{T}\right]=\int_{\mathbb{T}} H \Phi_{\mathbf{x y}} H^{*}
\end{aligned}
$$

where $H$ denotes the discrete-time Fourier transform of $h$ :

$$
H\left(e^{j \vartheta}\right)=\sum_{t \geq 0} h_{t} e^{-j \vartheta t} .
$$

We can rewrite the objective function in 11 in terms of $\Phi$ :

$$
\begin{aligned}
& \mathbb{E}\left[\left\|(h \star(\mathbf{x}-\mathbf{y}))_{t}\right\|^{2}\right]=\operatorname{tr} \mathbb{E}\left[(h \star(\mathbf{x}-\mathbf{y}))_{t}(h \star(\mathbf{x}-\mathbf{y}))_{t}^{T}\right] \\
& =\operatorname{tr} \mathbb{E}[(h \star \mathbf{x}))_{t}(h \star \mathbf{x})_{t}^{T}+(h \star \mathbf{y})_{t}(h \star \mathbf{y})_{t}^{T} \\
& \left.\quad-(h \star \mathbf{x})_{t}(h \star \mathbf{y})_{t}^{T}-(h \star \mathbf{y})_{t}(h \star \mathbf{x})_{t}^{T}\right] \\
& =\operatorname{tr} \int_{\mathbb{T}} H\left(\Phi_{\mathbf{x}}+\Phi_{\mathbf{y}}-\Phi_{\mathbf{x y}}-\Phi_{\mathbf{y} \mathbf{x}}\right) H^{*} \\
& =\operatorname{tr} \int_{\mathbb{T}} \Omega\left(\Phi_{\mathbf{x}}+\Phi_{\mathbf{y}}-\Phi_{\mathbf{x y}}-\Phi_{\mathbf{y} \mathbf{x}}\right)
\end{aligned}
$$

where $\Omega\left(e^{j \vartheta}\right)=H\left(e^{j \vartheta}\right)^{*} H\left(e^{j \vartheta}\right)$. In what follows we assume that $\Omega, \Phi_{\mathbf{x}}, \Phi_{\mathbf{y}} \in \mathcal{S}_{m}^{+}$. The latter condition is not so restrictive. Indeed, it means those spectral densities admit a minimum phase spectral factor. Therefore, we can rewrite (1) in terms of $\Phi$ :

$$
\begin{array}{r}
d_{\Omega}\left(p_{\mathbf{x}}, p_{\mathbf{y}}\right)^{2}=\inf _{\Phi_{\mathbf{x} \mathbf{y}}} \operatorname{tr} \int_{\mathbb{T}} \Omega\left(\Phi_{\mathbf{x}}+\Phi_{\mathbf{y}}-\Phi_{\mathbf{x y}}-\Phi_{\mathbf{y x}}\right) \\
\text { s.t. }\left[\begin{array}{cc}
\Phi_{\mathbf{x}} & \Phi_{\mathbf{x y}} \\
\Phi_{\mathbf{y x}} & \Phi_{\mathbf{y}}
\end{array}\right] \geq 0
\end{array}
$$

where the infimization is only with respect to $\Phi_{\mathbf{x y}}$ because $\Phi_{\mathbf{x}}$ and $\Phi_{\mathbf{y}}$ have been already fixed by constraints 2 - 3 . Notice that we added the subscript $\Omega$ to $d$ in order to stress such a dependence. Since $\Phi_{\mathbf{y}} \in \mathcal{S}_{m}^{+}$, we have $\Phi_{\mathbf{y}}>0$. Accordingly, condition $\Phi \geq 0$ is equivalent to $\Phi_{\mathbf{x}}-\Phi_{\mathbf{x y}} \Phi_{\mathbf{y}}^{-1} \Phi_{\mathbf{y x}} \geq 0$ and hence

$$
\begin{aligned}
d_{\Omega}\left(p_{\mathbf{x}}, p_{\mathbf{y}}\right)^{2}= & \inf _{\Phi_{\mathbf{x} \mathbf{y}}} \operatorname{tr} \int_{\mathbb{T}} \Omega\left(\Phi_{\mathbf{x}}+\Phi_{\mathbf{y}}-\Phi_{\mathbf{x y}}-\Phi_{\mathbf{y x}}\right) \\
& \text { s.t. } \Phi_{\mathbf{x}}-\Phi_{\mathbf{x y}} \Phi_{\mathbf{y}}^{-1} \Phi_{\mathbf{y} \mathbf{x}} \geq 0 .
\end{aligned}
$$

Let

$$
\Lambda:=\Phi_{\mathbf{x}}-\Phi_{\mathbf{x y}} \Phi_{\mathbf{y}}^{-1} \Phi_{\mathbf{y x}}
$$

then

$$
\left(\Phi_{\mathbf{x}}-\Lambda\right)^{1 / 2} \Upsilon=\Phi_{\mathbf{x y}} \Phi_{\mathbf{y}}^{-1 / 2}
$$

where $\Upsilon$ is an all-pass function, i.e. $\Upsilon\left(e^{j \vartheta}\right) \Upsilon\left(e^{j \vartheta}\right)^{*}=I$ for any $e^{j \vartheta} \in \mathbb{T}$. Thus,

$$
\Phi_{\mathbf{x y}}=\left(\Phi_{\mathbf{x}}-\Lambda\right)^{1 / 2} \Upsilon \Phi_{\mathbf{y}}^{1 / 2}
$$

Substituting $\Phi_{\mathbf{x y}}$ with $\Lambda$ and $\Upsilon$ in $[6$ we obtain

$$
\begin{array}{r}
d_{\Omega}\left(p_{\mathbf{x}}, p_{\mathbf{y}}\right)^{2}-\int_{\mathbb{T}} \Omega\left(\Phi_{\mathbf{x}}+\Phi_{\mathbf{y}}\right)=\inf _{\Lambda} \mathcal{F}(\Lambda) \\
\text { s.t. } \Lambda \geq 0
\end{array}
$$

where

$$
\begin{gathered}
\mathcal{F}(\Lambda)=\inf _{\Upsilon} \operatorname{tr} \int_{\mathbb{T}}\left(\Psi \Upsilon+\Upsilon^{*} \Psi^{*}\right) \\
\text { s.t. } \Upsilon \Upsilon^{*}=I
\end{gathered}
$$

and

$$
\Psi=-\Phi_{\mathbf{y}}^{1 / 2} \Omega\left(\Phi_{\mathbf{x}}-\Lambda\right)^{1 / 2} .
$$

We solve problem $(8)$ by means of duality theory. The Lagrangian is

$$
L(\Upsilon, \Delta)=\operatorname{tr} \int_{\mathbb{T}}\left(\Psi \Upsilon+\Upsilon^{*} \Psi^{*}\right)+\operatorname{tr} \int_{\mathbb{T}}\left(\Upsilon \Upsilon^{*}-I\right) \Delta
$$

where $\Delta \in \mathbb{L}_{\infty}^{m \times m}(\mathbb{T})$ and such that $\Delta=\Delta^{*}$ is the Lagrange multiplier. Moreover, the first and the second variation of $L(\cdot, \Delta)$ along $\delta \Upsilon \in \mathbb{L}_{\infty}^{m \times m}(\mathbb{T})$ are

$$
\begin{aligned}
\delta L(\Upsilon, \Delta ; \delta \Upsilon) & =\operatorname{tr} \int_{\mathbb{T}} \Psi \delta \Upsilon+\delta \Upsilon^{*} \Psi^{*}+\delta \Upsilon \Upsilon^{*} \Delta+\Upsilon \delta \Upsilon^{*} \Delta \\
\delta^{2} L(\Upsilon, \Delta ; \delta \Upsilon) & =2 \operatorname{tr} \int_{\mathbb{T}} \delta \Upsilon^{*} \Delta \delta \Upsilon .
\end{aligned}
$$

Accordingly, $L$ is lower bounded if and only if $\Delta \geq 0$. Moreover, if $\Delta>0$ then $L$ is strictly convex with respect to $\Upsilon$ and the unique point of minimum is given by the stationarity condition $\delta L(\Upsilon, \Delta ; \delta \Upsilon)=0$ for any $\delta \Upsilon \in \mathbb{L}_{\infty}^{m \times m}(\mathbb{T})$ which implies the optimal form

$$
\Upsilon^{\circ}=-\Delta^{-1} \Psi^{*}
$$

Accordingly, the dual functional is

$$
\mathcal{J}(\Delta)=L\left(\Upsilon^{\circ}, \Delta\right)=-\operatorname{tr} \int_{\mathbb{T}} \Psi \Delta^{-1} \Psi^{*}+\Delta
$$

and the dual problem is $\max _{\Delta>0} J(\Delta)$. Notice that the first and the second variation along $\delta \Delta \in \mathbb{L}_{\infty}^{m}(\mathbb{T})$ are

$$
\begin{aligned}
\delta \mathcal{J}(\Delta ; \delta \Delta) & =\operatorname{tr} \int_{\mathbb{T}} \Psi \Delta^{-1} \delta \Delta \Delta^{-1} \Psi^{*}+\delta \Delta \\
\delta^{2} \mathcal{J}(\Delta ; \delta \Delta) & =-2 \operatorname{tr} \int_{\mathbb{T}} \Psi \Delta^{-1} \delta \Delta^{-1} \delta \Delta \Delta^{-1} \Psi^{*} .
\end{aligned}
$$

Under the assumption that $\Delta>0$ and $\Psi$ is full rank almost everywhere, we have that $\delta^{2} \mathcal{J}(\Delta ; \delta \Delta)<0$ for any $\delta \Delta \in \mathbb{L}_{\infty}^{m \times m}(\mathbb{T})$ and $\delta \Delta \neq 0$. Accordingly, $\mathcal{J}$ is strictly concave, and the unique point of minimum is given by setting equal to zero its first variation in any direction which gives

$$
\Delta^{2}=\Psi^{*} \Psi
$$

Therefore the point of maximum is

$$
\Delta^{\circ}=\left(\Psi^{*} \Psi\right)^{1 / 2} \text {. }
$$


Notice that $\Delta^{\circ}>0$ (i.e. our assumption on $\Delta$ is satisfied) provided that $\Psi$ is full rank almost everywhere. In this case, the unique solution to 8 is

$$
\Upsilon^{\circ}=-\left(\Delta^{\circ}\right)^{-1} \Psi^{*}=-\left(\Psi^{*} \Psi\right)^{-1 / 2} \Psi^{*} .
$$

Moreover, it is not difficult to see that

$$
\Psi \Upsilon^{\circ}=-\left(\Psi \Psi^{*}\right)^{1 / 2}=-\left(\Phi_{\mathbf{y}}^{1 / 2} \Omega\left(\Phi_{\mathbf{x}}-\Lambda\right) \Omega \Phi_{\mathbf{y}}^{1 / 2}\right)^{1 / 2}
$$

where we have exploited (9). Substituting (11) in (8) we obtain:

$$
\mathcal{F}(\Lambda)=2 \operatorname{tr} \int_{\mathbb{T}} \Psi \Upsilon^{\circ}=-2 \operatorname{tr} \int_{\mathbb{T}}\left(\Phi_{\mathbf{y}}^{1 / 2} \Omega\left(\Phi_{\mathbf{x}}-\Lambda\right) \Omega \Phi_{\mathbf{y}}^{1 / 2}\right)^{1 / 2} .
$$

Accordingly, the optimal solution to 7 is $\Lambda^{\circ}=0$ and thus:

$$
d_{\Omega}\left(p_{\mathbf{x}}, p_{\mathbf{y}}\right)^{2}=\operatorname{tr} \int_{\mathbb{T}} \Omega \Phi_{\mathbf{x}}+\Omega \Phi_{\mathbf{y}}-2\left(\Phi_{\mathbf{y}}^{1 / 2} \Omega \Phi_{\mathbf{x}} \Omega \Phi_{\mathbf{y}}^{1 / 2}\right)^{1 / 2}
$$

which gives 4 . Notice that $\Psi=-\Phi_{\mathbf{y}}^{1 / 2} \Omega \Phi_{\mathbf{x}}^{1 / 2}$ is full rank almost everywhere for $\Lambda^{\circ}=0$, i.e. our assumption on $\Psi$ is satisfied.

Proposition 2.2: For any square spectral factor $W_{\mathbf{x}}$ of $\Phi_{\mathbf{x}}$, we have

$$
\begin{aligned}
& d_{\Omega}\left(p_{\mathbf{x}}, p_{\mathbf{y}}\right)^{2}=\min \left\{\int_{\mathbb{T}}\left\|W_{\mathbf{x}}-W_{\mathbf{y}}\right\|_{\Omega}^{2}\right. \text { s.t. } \\
& \left.\qquad W_{\mathbf{y}} \in \mathbb{L}_{\infty}^{m \times m}(\mathbb{T}), W_{\mathbf{y}} W_{\mathbf{y}}^{*}=\Phi_{\mathbf{y}}\right\} .
\end{aligned}
$$

Moreover, $d_{\Omega}\left(p_{\mathbf{x}}, p_{\mathbf{y}}\right)$ is a bona fide distance function.

Proof: Once $W_{\mathbf{x}}$ is fixed, any square spectral factor $W_{\mathbf{x}}^{\prime}$ of $\Phi_{\mathbf{x}}$ can be written as $W_{\mathbf{x}}^{\prime}=W_{\mathbf{x}} \Upsilon$ where $\Upsilon \in \mathbb{L}_{\infty}^{m \times m}(\mathbb{T})$ is an all-pass function. Therefore, we have

$$
\begin{array}{r}
\operatorname{tr} \int_{\mathbb{T}}\left\|W_{\mathbf{x}}^{\prime}-W_{\mathbf{y}}\right\|_{\Omega}^{2}=\operatorname{tr} \int_{\mathbb{T}}\left(W_{\mathbf{x}}^{\prime}-W_{\mathbf{y}}\right)^{*} \Omega\left(W_{\mathbf{x}}^{\prime}-W_{\mathbf{y}}\right) \\
=\operatorname{tr} \int_{\mathbb{T}}\left(W_{\mathbf{x}}-W_{\mathbf{y}} \Upsilon^{*}\right)^{*} \Omega\left(W_{\mathbf{x}}-W_{\mathbf{y}} \Upsilon^{*}\right)
\end{array}
$$

and $W_{\mathbf{y}} \Upsilon^{*}$ is a spectral factor a $\Phi_{\mathbf{y}}$. Therefore, the right hand side of (12) does not depend on the particular choice of the square spectral factor for $\Phi_{\mathbf{x}}$. To prove the equality in $(12)$, observe that the right hand side can be rewritten as

$$
\min \left\{\int_{\mathbb{T}}\left\|W_{\mathbf{x}}-\Phi_{\mathbf{y}}^{1 / 2} \Upsilon\right\|_{\Omega}^{2}, \Upsilon \in \mathbb{L}_{\infty}^{m \times m}(\mathbb{T}), \Upsilon \Upsilon^{*}=I\right\} .
$$

The corresponding Lagrangian is

$$
L(\Upsilon, \Lambda)=\int_{\mathbb{T}}\left\|W_{\mathbf{x}}-\Phi_{\mathbf{y}}^{1 / 2} \Upsilon\right\|_{\Omega}^{2}+\operatorname{tr}\left(\Upsilon \Upsilon^{*}-I\right) \Lambda
$$

where $\Lambda=\Lambda^{*} \in \mathbb{L}_{\infty}^{m \times m}(\mathbb{T})$ is the Lagrange multiplier. Under the assumption that $\Lambda>0$, the minimum of $L$ with respect to $\Upsilon$ is $\Upsilon(\Lambda)=\Lambda^{-1} \Phi_{\mathbf{y}}^{1 / 2} \Omega W_{\mathbf{x}}$. It is not difficult to see that the Lagrange multiplier satisfying the constraint in the primal problem is $\Lambda^{\circ}=$ $\Phi_{\mathbf{y}}^{1 / 2} \Omega \Phi_{\mathbf{x}} \Omega \Phi_{\mathbf{y}}^{1 / 2}>0$. Therefore, the minimum point of the right hand side in (12) is

$$
W_{\mathbf{y}}^{\circ}=\Phi_{\mathbf{y}}^{1 / 2}\left(\Phi_{\mathbf{y}}^{1 / 2} \Omega \Phi_{\mathbf{x}} \Omega \Phi_{\mathbf{y}}^{1 / 2}\right)^{-1 / 2} \Phi_{\mathbf{y}}^{1 / 2} \Omega W_{\mathbf{x}}
$$

and by direct substitution we obtain (4). The last part of the claim can be easily checked.

In the special case that $\Omega=I$, we obtain the Hellinger distance for multivariate power spectral densities [6]:

$$
\begin{aligned}
d\left(p_{\mathbf{x}}, p_{\mathbf{y}}\right):= & {\left[\operatorname { i n f } \left\{\int_{\mathbb{T}}\left\|W_{\mathbf{x}}-W_{\mathbf{y}}\right\|^{2}\right.\right. \text { s.t. }} \\
& \left.\left.W_{\mathbf{y}} \in \mathbb{L}_{\infty}^{m \times m}(\mathbb{T}), W_{\mathbf{y}} W_{\mathbf{y}}^{*}=\Phi_{\mathbf{y}}\right\}\right]^{1 / 2} .
\end{aligned}
$$

Accordingly, $d_{\Omega}\left(p_{\mathbf{x}}, p_{\mathbf{y}}\right)$ is the weighted Hellinger distance with weight the matricial function $\Omega \in \mathcal{S}_{m}^{+}$. Finally, notice that $d_{\Omega}\left(p_{\mathbf{x}}, p_{\mathbf{y}}\right)$ is completely characterized by $\Phi_{\mathbf{x}}$ and $\Phi_{\mathbf{y}}$. Accordingly, in what

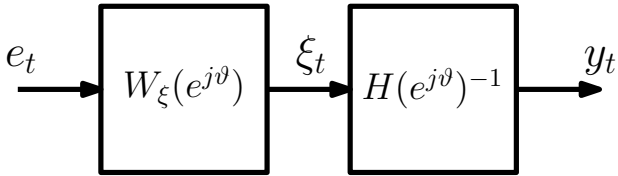

Fig. 1. Generative model for processes $y$ and $\xi$.

follows we will use the notation $d_{\Omega}\left(\Phi_{\mathbf{x}}, \Phi_{\mathbf{y}}\right)$ to denote the weighted transportation distance between the Gaussian processes having power spectral density $\Phi_{\mathbf{x}}$ and $\Phi_{\mathbf{y}}$, respectively.

\section{SPECTRAL Estimation WITH INDIRECT MEASUREMENTS}

In this section we show how the weighted Hellinger distance can be used to estimate stochastic processes from indirect measurements. As we will see, the appealing property is that the corresponding estimator accounts for the transfer matrix characteristic of the sensor network. Consider two $m$-dimensional zero mean Gaussian stationary stochastic processes $\mathbf{y}$ and $\boldsymbol{\xi}$ generated by a normalized white Gaussian noise process e as depicted in Figure 1] We assume that $H$, defined in (5), is a rational transfer matrix, causally invertible and known. The transfer matrix $W_{\boldsymbol{\xi}}$ is such that $\Phi_{\boldsymbol{\xi}}=W_{\boldsymbol{\xi}} W_{\boldsymbol{\xi}}^{*} \in \mathcal{S}_{m}^{+}$and it is not known. It is worth noting that the spectral density of $\mathbf{y}$ is $\Phi_{\mathbf{y}}=H^{-1} \Phi_{\boldsymbol{\xi}} H^{-*} \in \mathcal{S}_{m}^{+}$while the one of $\boldsymbol{\xi}$ is $\Phi_{\boldsymbol{\xi}}$. Next, we face the problem to find an estimate of $\Phi_{\xi}$ from a finite length realization $y^{N}=\left\{y_{1} \ldots y_{N}\right\}$ of $\mathbf{y}$. In plain words, we want to perform a multivariate spectral estimation task from indirect measurements: we have a network of $m$ sensors modeled by $\mathbf{y}$ and measuring indirectly $m$ variables of interest modeled by $\boldsymbol{\xi}$. Moreover, $H^{-1}$ is the transfer matrix characteristic of the sensor network: $\left[H^{-1}\right]_{i l}$ denotes the transfer function between the $l$-th variable of interest and the $i$-th sensor.

We address such a problem using a THREE-like framework. Suppose that a power spectral density (called prior) $\Psi_{\boldsymbol{\xi}} \in \mathcal{S}_{m}^{+}$, which gives some a priori information on $\Phi_{\xi}$, is available. If no prior is available we choose the uninformative prior $\Psi_{\xi}=I$, i.e. the spectral density corresponding to white noise. Then, we fix a bank of filters

$$
G(z)=(z I-A)^{-1} B
$$

where $A \in \mathbb{R}^{n \times n}$ is a stability matrix, $B \in \mathbb{R}^{n \times m}$ is full column rank with $n>m$, and $(A, B)$ is a reachable pair. Let $\Sigma$ denote the steady state covariance of the output of the bank of filters

$$
x_{t+1}=A x_{t}+B y_{t} .
$$

We compute an estimate $\hat{\Sigma}$, based on the data $y^{N}$, of $\Sigma$. Then, an estimate of $\Phi_{\xi}$ is given by the spectrum approximation problem:

$$
\begin{aligned}
\hat{\Phi}_{\boldsymbol{\xi}, T}=\underset{\Phi_{\boldsymbol{\xi}} \in \mathcal{S}_{m}^{+}}{\operatorname{argmax}} d\left(\Phi_{\boldsymbol{\xi}}, \Psi_{\boldsymbol{\xi}}\right) \\
\text { s.t. } \Phi=H^{-1} \Phi_{\boldsymbol{\xi}} H^{-*}, \int_{\mathbb{T}} G \Phi G^{*}=\hat{\Sigma}
\end{aligned}
$$

where "T" stand for transportation distance. The latter problem can be rewritten only in terms of $\Phi$ :

$$
\begin{array}{r}
\hat{\Phi}=\underset{\Phi \in \mathcal{S}_{m}^{+}}{\operatorname{argmax}} d_{\Omega}(\Phi, \Psi) \\
\text { s.t. } \int_{\mathbb{T}} G \Phi G^{*}=\hat{\Sigma}
\end{array}
$$

where $\hat{\Phi}_{\boldsymbol{\xi}, T}=H \hat{\Phi} H^{*}, \Psi_{\boldsymbol{\xi}}=H \Psi H^{*}$ and $\Omega=H^{*} H$. In the special case that $G(z)=\left[z^{-l} I_{m} \ldots z^{-1} I_{m}\right]^{T}$, we have that $\Sigma$ is a $n \times n$, with $n=l m$, block Toeplitz matrix whose first block row is $\left[\Sigma_{0} \Sigma_{1} \ldots \Sigma_{n-1}\right]$ and $\Sigma_{k}=\mathbb{E}\left[y_{t} y_{t+k}^{T}\right]$. Therefore, with this 
particular choice of $G(z)$, we fix the first covariance lags of $\hat{\Phi}$, i.e. $\hat{\Phi}$ is the solution to a covariance extension problem. It is worth noting $\hat{\Sigma}$ must guarantee the feasibility of Problem $\sqrt{14}$ that is there exits at least one $\tilde{\Phi} \in \mathcal{S}_{m}^{+}$such that $\int_{\mathbb{T}} G \tilde{\Phi} G^{*}=\Sigma$. In [8], it has been shown that $\hat{\Sigma}$ guarantees the feasibility of the problem if and only if $\hat{\Sigma}>0$ and $\hat{\Sigma} \in \operatorname{Range} \Gamma$ where $\Gamma$ is the linear operator defined as

$$
\begin{aligned}
\Gamma: \mathcal{C}_{m}(\mathbb{T}) \rightarrow \mathcal{Q}_{n} \\
\Phi \mapsto \int_{\mathbb{T}} G \Phi G^{*}
\end{aligned}
$$

where $\mathcal{C}_{m}(\mathbb{T})$ denotes the family of $\mathbb{C}^{m \times m}$ and hermitian-valued continuous functions on the unit circle $\mathbb{T}$. Optimization approaches have proposed to compute such an estimate from data, see [27], [7].

Let $W_{\Psi}$ and $W$ be a square spectral factor of $\Psi$ and $\Phi$, respectively, then Problem [14] is equivalent to

$$
\begin{gathered}
\hat{W}=\underset{W \in \mathbb{L}_{\infty}^{m \times m}(\mathbb{T})}{\operatorname{argmax}} \operatorname{tr} \int_{\mathbb{T}}\left(W-W_{\Psi}\right)\left(W-W_{\Psi}\right)^{*} \Omega \\
\text { s.t. } \int_{\mathbb{T}} G W W^{*} G^{*}=\hat{\Sigma} .
\end{gathered}
$$

The Lagrangian of Problem 15 is

$$
\begin{aligned}
L(W, \Lambda)= & \operatorname{tr} \int_{\mathbb{T}}\left(W-W_{\Psi}\right)\left(W-W_{\Psi}\right)^{*} \Omega \\
& +\operatorname{tr}\left(\Lambda \int_{\mathbb{T}} G W W^{*} G^{*}\right)-\operatorname{tr}(\Lambda \hat{\Sigma}) \\
= & \operatorname{tr} \int_{\mathbb{T}}\left(\Omega+G^{*} \Lambda G\right) W W^{*}-\Omega W_{\Psi} W^{*}-\Omega W W_{\Psi}^{*} \\
& +\Omega W_{\Psi} W_{\Psi}^{*}-\operatorname{tr}(\Lambda \hat{\Sigma})
\end{aligned}
$$

where $\Lambda \in \mathcal{Q}_{n}$ is the Lagrange multiplier. $L(\cdot, \Lambda)$ is bounded below only if $\Omega+G^{*} \Lambda G>0$. In this case the infimum is attained and given by setting its first variation equal to zero

$$
\begin{aligned}
\delta L(W, \Lambda ; \delta W)= & \int_{\mathbb{T}}\left(\Omega+G^{*} \Lambda G\right) \delta W W^{*}+\left(\Omega+G^{*} \Lambda G\right) W \delta W^{*} \\
& -\Omega W_{\Psi} \delta W^{*}-\Omega \delta W W_{\Psi}^{*}=0
\end{aligned}
$$

for any $\delta W \in \mathbb{L}_{\infty}^{m \times m}(\mathbb{T})$. The latter leads to the optimality condition

$$
\left(\Omega+G^{*} \Lambda G\right) W-\Omega W_{\Psi}=0
$$

and thus the optimal form of $W$ is

$$
W^{\circ}=\left(\Omega+G^{*} \Lambda G\right)^{-1} \Omega W_{\Psi} .
$$

Therefore, the dual problem consists in finding a point of maximum $\Lambda^{\circ}$ for

$$
L\left(W^{\circ}, \Lambda\right)=-\left[\operatorname{tr} \int_{\mathbb{T}} \Omega \Psi \Omega\left(\Omega+G^{*} \Lambda G\right)^{-1}-\Omega \Psi\right]-\operatorname{tr}(\hat{\Sigma} \Lambda)
$$

over the set

$$
\mathcal{L}_{+}=\left\{\Lambda \in \mathcal{Q}_{n} \text { s.t. } \Omega+G^{*} \Lambda G>0\right\} .
$$

Thus, such a problem is equivalent to minimize

$$
J(\Lambda)=\operatorname{tr} \int_{\mathbb{T}} \Omega \Psi \Omega\left(\Omega+G^{*} \Lambda G\right)^{-1}+\operatorname{tr}(\hat{\Sigma} \Lambda)
$$

with $\Lambda \in \mathcal{L}_{+}$. As it has been shown in [6], $G^{*} \Lambda G=0$ for any $\Lambda \in$ [Range $\Gamma]^{\perp}$. Thus, $J\left(\Lambda+\Lambda_{\perp}\right)=J(\Lambda)$ for any $\Lambda_{\perp} \in[\text { Range } \Gamma]^{\perp}$. Accordingly, we can restrict the search of $\Lambda^{\circ}$ over the set $\mathcal{L}_{\Gamma,+}=$ $\mathcal{L}_{+} \cap$ Range $\Gamma$. Accordingly, we obtain the equivalent problem

$$
\Lambda^{\circ}=\underset{\Lambda \in \mathcal{L}_{\Gamma,+}}{\operatorname{argmin}} J(\Lambda) .
$$

Theorem 3.1: Problem (17) admits a unique solution.
Proof: Following arguments similar to the ones in [6 Lemma 7.5], it is possible to prove that $J$ is strictly convex on $\mathcal{L}_{\Gamma,+}$. Accordingly, if Problem (17) admits solution, then the latter is unique.

Next, we show the existence of such solution. First, notice that the set $\mathcal{L}_{\Gamma,+}$ is nonempty, indeed $0 \in \Lambda_{\Gamma,+}$, open and unbounded. Moreover, $J$ is a continuous function on $\mathcal{L}_{\Gamma,+}$. The idea is to show that Problem 17 is equivalent to minimize $J$ on a compact set, say $\mathcal{L}_{\Gamma,+}^{\star}$. Then, by the Weierstrass Theorem we conclude that the minimum exists.

We proceed to characterize $\mathcal{L}_{\Gamma,+}^{\star}$. First, we show that $J$ is bounded below on $\mathcal{L}_{\Gamma,+}$ :

$$
\begin{aligned}
J(\Lambda) & =\operatorname{tr} \int_{\mathbb{T}} \Psi^{1 / 2} \Omega\left(\Omega+G^{*} \Lambda G\right)^{-1} \Omega \Psi^{1 / 2}+\operatorname{tr}(\hat{\Sigma} \Lambda) \\
& \geq \operatorname{tr}(\hat{\Sigma} \Lambda)=\operatorname{tr} \int_{\mathbb{T}} G \tilde{\Phi} G^{*} \Lambda=\operatorname{tr} \int_{\mathbb{T}} \tilde{\Phi}^{1 / 2} G^{*} \Lambda G \tilde{\Phi}^{1 / 2} \\
& =\operatorname{tr} \int_{\mathbb{T}} \tilde{\Phi}^{1 / 2}\left(\Omega+G^{*} \Lambda G\right) \tilde{\Phi}^{1 / 2}-\operatorname{tr} \int_{\mathbb{T}} \tilde{\Phi}^{1 / 2} \Omega \tilde{\Phi}^{1 / 2} \\
& \geq-\int_{\mathbb{T}} \tilde{\Phi}^{1 / 2} \Omega \tilde{\Phi}^{1 / 2}>-\infty
\end{aligned}
$$

where we exploited the following facts: $\Omega\left(e^{j \vartheta}\right)+G\left(e^{j \vartheta}\right)^{*} \Lambda G\left(e^{j \vartheta}\right)$ is positive definite for any $e^{j \vartheta} \in \mathbb{T} ; \Omega$ and $\tilde{\Phi}$ are bounded and coercive. Following arguments similar to the ones in [6, Theorem 7.7], it is possible to prove that

$$
\begin{aligned}
\lim _{\Lambda \rightarrow \partial \mathcal{L}_{\Gamma,+}} J(\Lambda) & =\infty \\
\lim _{\|\Lambda\| \rightarrow \infty} J(\Lambda) & =\infty
\end{aligned}
$$

where $\partial \mathcal{L}_{\Gamma,+}$ denotes the boundary of $\mathcal{L}_{\Gamma,+}$ that is the set of $\Lambda \in$ $\mathcal{L}_{\Gamma,+}$ such that $\Omega\left(e^{j \bar{\vartheta}}\right)+G\left(e^{j \bar{\vartheta}}\right)^{*} \Lambda G\left(e^{j \bar{\vartheta}}\right) \geq 0$ and singular for at least one $e^{j \bar{\vartheta}} \in \mathbb{T}$. Accordingly, we can restrict the search of the minimum point of $J$ on the bounded and closed (and thus compact) set $\mathcal{L}_{\Gamma,+}^{\star}=\left\{\Lambda \in \mathcal{L}_{\Gamma,+}\right.$ s.t. $\left.\Omega+G^{*} \Lambda G \geq \alpha I,\|\Lambda\| \leq \beta\right\}$ for some $\alpha, \beta>0$.

Corollary 3.1: Problem [14) admits the unique solution

$$
\hat{\Phi}_{T}=\left(\Omega+G^{*} \Lambda^{\circ} G\right)^{-1} \Omega \Psi \Omega\left(\Omega+G^{*} \Lambda^{\circ} G\right)^{-1}
$$

where $\Lambda^{\circ}$ is the unique solution to 17 .

Proof: Recall that the optimal solution $\Lambda^{\circ}$ of 17 is a stationary point for $J$. The latter condition implies that $W^{\circ}$ in 16 with $\Lambda=$ $\Lambda^{\circ}$ is such that the spectral density $W^{\circ}\left(W^{\circ}\right)^{*}$ satisfies the moment constraints in 15. Accordingly, the duality gap is equal to zero. Hence, Problem 14 admits the unique solution $\hat{\Phi}_{T}=W^{\circ}\left(W^{\circ}\right)^{*}$ with $\Lambda=\Lambda^{\circ}$.

Since $\Omega=H^{*} H$ and $H$ is causally invertible, then 18$]$ can be rewritten as

$$
\begin{aligned}
\hat{\Phi}_{T}=H^{-1} & \left(I+H^{-*} G^{*} \Lambda^{\circ} G H^{-1}\right)^{-1} \Psi_{\xi} \\
& \times\left(I+H^{-*} G^{*} \Lambda^{\circ} G H^{-1}\right)^{-1} H^{-*}
\end{aligned}
$$

and thus

$$
\hat{\Phi}_{\boldsymbol{\xi}, T}=\left(I+H^{-*} G^{*} \Lambda^{\circ} G H^{-1}\right)^{-1} \Psi_{\boldsymbol{\xi}}\left(I+H^{-*} G^{*} \Lambda^{\circ} G H^{-1}\right)^{-1} .
$$

An alternative to Problem 13 is to consider

$$
\begin{aligned}
\hat{\Phi}_{\boldsymbol{\xi}, I S}=\underset{\Phi_{\boldsymbol{\xi}} \in \mathcal{S}_{m}^{+}}{\operatorname{argmax}} d_{I S}\left(\Phi_{\boldsymbol{\xi}} \| \Psi_{\boldsymbol{\xi}}\right) \\
\text { s.t. } \Phi=H^{-1} \Phi_{\boldsymbol{\xi}} H^{-*}, \int_{\mathbb{T}} G \Phi G^{*}=\hat{\Sigma}
\end{aligned}
$$

where $d_{I S}$ is the Itakura-Saito distance:

$$
d_{I S}\left(\Psi_{\boldsymbol{\xi}} \| \Phi_{\boldsymbol{\xi}}\right)=\operatorname{tr} \int_{\mathbb{T}}\left(\log \left|\Psi_{\boldsymbol{\xi}}\right|-\log \left|\Phi_{\boldsymbol{\xi}}\right|+\Phi_{\boldsymbol{\xi}} \Psi_{\boldsymbol{\xi}}^{-1}-I_{m}\right) .
$$


On the other hand, it is not difficult to see that

$$
d_{I S}\left(\Psi_{\xi} \| \Phi_{\xi}\right)=d_{I S}\left(H^{-1} \Psi_{\xi} H^{-*} \| H^{-1} \Phi_{\xi} H^{-*}\right)=d_{I S}(\Psi \| \Phi) .
$$

Accordingly, Problem (19) is equivalent to solve the THREE-like estimator using direct measurements proposed in [5]:

$$
\begin{array}{r}
\hat{\Phi}_{I S}=\underset{\Phi \in \mathcal{S}_{m}^{+}}{\operatorname{argmax}} d_{I S}(\Phi \| \Psi) \\
\text { s.t. } \int_{\mathbb{T}} G \Phi G^{*}=\hat{\Sigma}
\end{array}
$$

where $\hat{\Phi}_{\boldsymbol{\xi}, I S}=H \hat{\Phi}_{I S} H^{*}$. The optimal solution of 20 is

$$
\begin{aligned}
\hat{\Phi}_{I S} & =\left(\Psi^{-1}+G^{*} \Lambda G\right)^{-1} \\
& =H^{-1}\left(\Psi_{\xi}^{-1}+H^{-*} G^{*} \Lambda G H^{-1}\right)^{-1} H^{-*}
\end{aligned}
$$

where $\Lambda$ is given by solving the corresponding dual problem, see [5]. Hence, we have

$$
\hat{\Phi}_{\boldsymbol{\xi}, I S}=\left(\Psi_{\xi}^{-1}+H^{-*} G^{*} \Lambda G H^{-1}\right)^{-1} .
$$

The fact that the objective function in 20 is the same for the cases
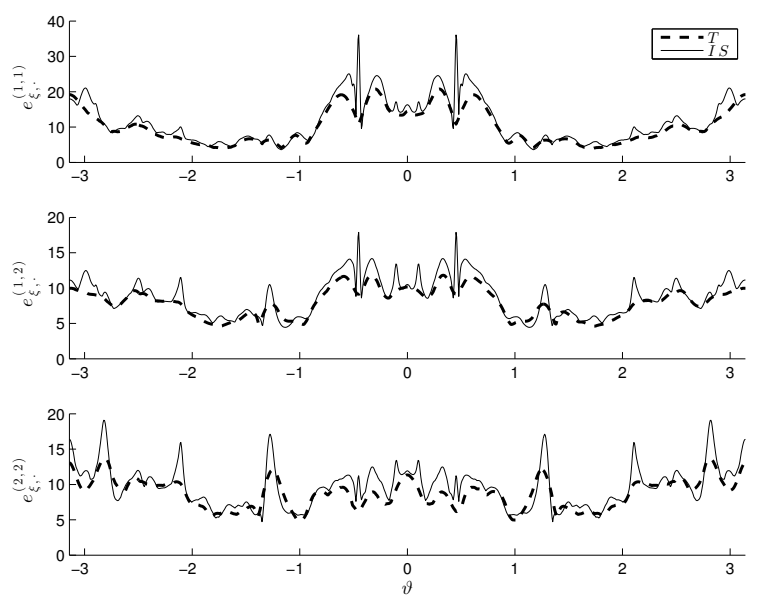

Fig. 2. Average errors in the estimation of $\Phi_{\boldsymbol{\xi}}$ using the estimator with the transportation distance (T) and the Itakura-Saito distance (IS).

of indirect/direct measurements reveals that the Itakura-Saito distance does not adapt the optimization scheme for a possible improvement in the case of indirect measurements. Drawing inspiration from (14, we could consider the weighted Itakura-Saito distance, see [25], with weight function $\Omega$ :

$$
\begin{array}{r}
\hat{\Phi}=\underset{\Phi \in \mathcal{S}_{m}^{+}}{\operatorname{argmax}} d_{I S, \Omega}(\Phi, \Psi) \\
\text { s.t. } \int_{\mathbb{T}} G \Phi G^{*}=\hat{\Sigma}
\end{array}
$$

where

$$
d_{I S, \Omega}(\Psi \| \Phi)=\operatorname{tr} \int_{\mathbb{T}} \Omega\left(\log |\Psi|-\log |\Phi|+W_{\Psi}^{-1} \Phi W_{\Psi}^{-*}-I_{m}\right)
$$

and $W_{\Psi}$ is a spectral factor of $\Psi$. It is not difficult to see that $d_{I S, \Omega}(\Psi \| \Phi) \geq 0$ and equality holds if and only if $\Phi=\Psi$. Moreover, $d_{I S, \Omega}(\Psi \| \Phi)$ is strictly convex with respect to $\Phi$. Notice that for $\Omega=I$ we obtain Itakura-Saito distance. The Lagrangian of Problem 22, is

$$
\begin{aligned}
\mathcal{L}(\Phi, \Lambda)=\operatorname{tr} & {\left[\int_{\mathbb{T}} \Omega\left(\log |\Psi|-\log |\Phi|+W_{\Psi}^{-1} \Phi W_{\Psi}^{-*}-I_{m}\right)\right.} \\
& \left.+G^{*} \Lambda G \Phi\right]-\operatorname{tr}(\hat{\Sigma} \Lambda)
\end{aligned}
$$

which is bounded below only if $W_{\Psi}^{-*} \Omega W_{\Psi}^{-1}+G^{*} \Lambda G>0$. Moreover, it is not difficult to prove that $\mathcal{L}$ is strictly convex with respect to $\Phi$ and its point of minimum is given by setting equal to zero the first variation for any $\delta \Phi \in \mathbb{L}_{\infty}^{m \times m}(\mathbb{T})$

$$
\begin{aligned}
\delta \mathcal{L}(\Phi, \Lambda ; \delta \Phi)=\operatorname{tr} & {\left[\int_{\mathbb{T}} \Omega W_{\Psi}^{-1} \delta \Phi W_{\Psi}^{-*}+G^{*} \Lambda G \delta \Phi\right.} \\
& \left.\left.-\Omega \int_{0}^{\infty}(\Phi+t I)^{-1} \delta \Phi(\Phi+t I)^{-1}\right) \mathrm{~d} t\right]
\end{aligned}
$$

leading to the optimality condition

$$
\int_{0}^{\infty}(\Phi+t I)^{-1} \Omega(\Phi+t I)^{-1} \mathrm{~d} t=W_{\Psi}^{-*} \Omega W_{\Psi}^{-1}+G^{*} \Lambda G .
$$

Clearly, it is not possible to find an explicit form for the optimal $\Phi$ and thus the dual analysis cannot carried out. Accordingly, we are not able to solve Problem 22. On the other hand, in the special case that $\Omega=\omega I$ with $\omega \in \mathcal{S}_{1}^{+}$, condition 25 becomes

$$
\Phi=\left(\Psi^{-1}+\omega^{-1} G^{*} \Lambda G\right)^{-1}
$$

and the dual problem is equivalent to solve

$$
\begin{aligned}
& \Lambda^{\circ}=\underset{\Lambda}{\operatorname{argmin}}-\int_{\mathbb{T}} \omega \log \left|\Psi^{-1}+\omega^{-1} G^{*} \Lambda G\right|+\operatorname{tr}(\hat{\Sigma} \Lambda) \\
& \text { s.t. } \Psi^{-1}+\omega^{-1} G^{*} \Lambda G>0 .
\end{aligned}
$$

Moreover, it is possible to prove that Problem (27) admits a solution and the solution to the primal problem is 26 with $\Lambda=\Lambda^{\circ}$.

\section{SIMULATION RESULTS}

We test the spectral estimator with indirect measurements using the weighted transportation distance. More precisely, we consider a Monte Carlo study constituted by 50 experiments. In each experiment:

- We generate a bivariate process $\xi$ with spectral density $\Phi_{\xi}=$ $W_{\boldsymbol{\xi}} W_{\boldsymbol{\xi}}^{*} \in \mathcal{S}_{2}^{+}, W_{\boldsymbol{\xi}}(z)=C_{\boldsymbol{\xi}}\left(z I-A_{\boldsymbol{\xi}}\right)^{-1} B_{\boldsymbol{\xi}}+D_{\boldsymbol{\xi}}$ and $A_{\boldsymbol{\xi}}, B_{\boldsymbol{\xi}}$, $C_{\xi}, D_{\xi}$ are randomly generated. In particular, $A_{\xi}$ is a $4 \times 4$ matrix whose eigenvalues are with absolute value less than or equal to 0.8 .

- We generate the transfer matrix characteristic $H(z)^{-1}=$ $C_{H}\left(z I-A_{H}\right)^{-1} B_{H}+D_{H}$. Matrices $A_{H}, B_{H}, C_{H}, D_{H}$ are randomly generated and such that $H(z)^{-1}$ is causally invertible. Moreover, $A_{H}$ is $4 \times 4$ matrix whose eigenvalues are with absolute value less than or equal to 0.7 .

- We generate $\Psi_{\boldsymbol{\xi}}=W_{p} W_{p}^{*}, W_{p}(z)=C_{p}\left(z I-A_{p}\right)^{-1} B_{p}+D_{p}$, $A_{p}=A_{\boldsymbol{\xi}}+\delta A, B_{p}=B_{\boldsymbol{\xi}}+\delta B, C_{p}=C_{\boldsymbol{\xi}}+\delta C, D_{p}=D_{\boldsymbol{\xi}}+$ $\delta D$. The perturbations matrices $\delta A, \delta B, \delta C, \delta D$ are randomly generated such that: its infinity matrix norm is equal to 0.08 , $A_{p}$ is Schur stable and $\Psi_{\boldsymbol{\xi}} \in \mathcal{S}_{2}^{+}$.

- We generate a short dataset $y(1) \ldots y(N)$, with $N=100$, extracted form a realization of $\mathbf{y}$ whose shaping filter is $H^{-1} W_{\boldsymbol{\xi}}$.

- We set the bank of filters as $G(z)=\left[z^{-l} I_{m} \ldots z^{-1} I_{m}\right]^{T}$ with $l=12$.

- We compute $\hat{\Sigma}$ from the data using the optimization procedure in [7] which guarantees the feasibility condition.

- We compute $\hat{\Phi}_{\xi, T}$ that is the solution to 13 .

- We compute the errors for each entry of the spectral density:

$$
e_{\boldsymbol{\xi}, T}^{(i, k)}(\vartheta)=\left|\left[\Phi_{\boldsymbol{\xi}}\left(e^{j \vartheta}\right)\right]_{i, k}-\left[\hat{\Phi}_{\boldsymbol{\xi}, T}\left(e^{j \vartheta}\right)\right]_{i, k}\right| .
$$

In Figure 2 we compare the average error defined in 28) as a function of $\vartheta$ in the Monte Carlo study and the one obtained using the estimator $\hat{\Phi}_{\boldsymbol{\xi}, I S}$ defined in 20 which uses the Itakura-Saito distance. Furthermore, the average value of the $L_{2}$ norm of the error between the estimated spectral density and $\Phi_{\xi}$ is equal to 30.58 using 
$\hat{\Phi}_{\xi, T}$ and 37.35 using $\hat{\Phi}_{\xi, I S}$. As we can see, the estimator equipped with the transportation distance performs better than the one with the Itakura-Saito distance. Since the McMillan degree of $\hat{\Phi}_{\xi, T}$ is larger than the one of $\hat{\Phi}_{\boldsymbol{\xi}, I S}$, as sanity check, we also considered $\hat{\Phi}_{\boldsymbol{\xi}, I S}$ with larger values of $l$ : we have obtained worse results than the ones with $l=12$. We conclude that the superiority of $\hat{\Phi}_{\boldsymbol{\xi}, T}$ is probably due by the fact that the corresponding optimization scheme is adapted according to the transfer matrix characteristic modeling the indirect measurements.

Finally, it is worth noting that the case of direct measurements, i.e. $H(z)=I$, has been analyzed in [5]. It has been shown that the estimator with the Itakura-Saito distance is better than the one with the Hellinger distance in the case that the shaping filter has some poles close to the unit circle.

\section{CONCLUSIONS}

In this technical note, we have introduced the weighted transportation distance between Gaussian stationary processes. We showed that the latter corresponds to a weighted version of the Hellinger distance between multivariate power spectral densities. Then, we have developed a spectral estimator with indirect measurements using the transportation distance. Finally, a simulation study showed that the proposed estimator performs better than the one using the ItakuraSaito distance. It is worth noting that non-Gaussian processes as well as non-stationary processes are characterized by their joint probability density. Accordingly, the proposed optimal transport formulation seems the natural way for a possible extension in the aforementioned scenarios.

\section{REFERENCES}

[1] C. Byrnes, T. Georgiou, and A. Lindquist. A new approach to spectral estimation: A tunable high-resolution spectral estimator. IEEE Trans. Signal Processing, 48:3189-3205, 2000.

[2] C. Byrnes, S. Gusev, and A. Lindquist. A convex optimization approach to the rational covariance extension problem. SIAM J. Optim., 37:211229, 1998.

[3] Y. Chen, T. Georgiou, L. Ning, and A. Tannenbaum. Matricial Wasserstein-1 distance. IEEE control systems letters, 1(1):14-19, 2017.

[4] F. Elvander, A. Jakobsson, and J. Karlsson. Interpolation and extrapolation of Toeplitz matrices via optimal mass transport. IEEE Transactions on Signal Processing, 66(20):5285-5298, 2018.

[5] A. Ferrante, C. Masiero, and M. Pavon. Time and spectral domain relative entropy: A new approach to multivariate spectral estimation. IEEE Trans. Autom. Control, 57:2561-2575, 2012.

[6] A. Ferrante, M. Pavon, and F. Ramponi. Hellinger versus KullbackLeibler multivariable spectrum approximation. IEEE Trans. Autom. Control, 53:954-967, 2008.

[7] A. Ferrante, M. Pavon, and M. Zorzi. A maximum entropy enhancement for a family of high-resolution spectral estimators. IEEE Transactions on Automatic Control, 57(2):318-329, 2012.

[8] T. Georgiou. The structure of state covariances and its relation to the power spectrum of the input. IEEE Transactions on Automatic Control, 47(7):1056-1066, 2002.

[9] T. Georgiou. Relative entropy and the multivariable multidimensional moment problem. IEEE Transactions on Information Theory, 52(3):1052-1066, 2006.

[10] T. Georgiou, J. Karlsson, and M. Takyar. Metrics for power spectra: an axiomatic approach. IEEE Transactions on Signal Processing, 57(3):859-867, 2008.

[11] T. Georgiou and A. Lindquist. Kullback-Leibler approximation of spectral density functions. IEEE Transactions on Information Theory, 49(11):2910-2917, 2003.

[12] L. Kantorovich. On the translocation of masses. Doklady) Acad. Sci. URSS (NS), 37:199-201, 1942.

[13] J. Karlsson and T. Georgiou. Uncertainty bounds for spectral estimation. IEEE Transactions on Automatic Control, 58(7):1659-1673, 2013.

[14] M. Knott and C. Smith. On the optimal mapping of distributions. Journal of Optimization Theory and Applications, 43(1):39-49, 1984.
[15] L. Ning, T. Georgiou, and A. Tannenbaum. On matrix-valued MongeKantorovich optimal mass transport. IEEE transactions on automatic control, 60(2):373-382, 2015.

[16] L. Ning, X. Jiang, and T. Georgiou. On the geometry of covariance matrices. IEEE Signal Processing Letters, 20(8):787-790, 2013.

[17] F. Ramponi, A. Ferrante, and M. Pavon. A globally convergent matricial algorithm for multivariate spectral estimation. IEEE Transactions on Automatic Control, 54(10):2376-2388, 2009.

[18] A. Ringh, J. Karlsson, and A. Lindquist. Multidimensional rational covariance extension with applications to spectral estimation and image compression. SIAM Journal on Control and Optimization, 54(4):19501982,2016

[19] A. Ringh, J. Karlsson, and A. Lindquist. Multidimensional rational covariance extension with approximate covariance matching. SIAM Journal on Control and Optimization, 56(2):913-944, 2018.

[20] B. Zhu. On the well-posedness of a parametric spectral estimation problem and its numerical solution. IEEE Transactions on Automatic Control, 65(3): 1089-1099, 2020.

[21] B. Zhu and G. Baggio. On the existence of a solution to a spectral estimation problem à la Byrnes-Georgiou-Lindquist. IEEE Transactions on Automatic Control, 64(2):820-825, 2018.

[22] B. Zhu, A. Ferrante, J. Karlsson, and M. Zorzi. M $^{2}$-spectral estimation: A relative entropy approach. Automatica (to appear), 2020.

[23] M. Zorzi. A new family of high-resolution multivariate spectral estimators. IEEE Transactions on Automatic Control, 59:892-904, 2014.

[24] M. Zorzi. Rational approximations of spectral densities based on the Alpha divergence. Mathematics of Control, Signals, and Systems, 26:259-278, 2014.

[25] M. Zorzi. An interpretation of the dual problem of the THREE-like approaches. Automatica, 62:87-92, 2015.

[26] M. Zorzi. Multivariate Spectral Estimation based on the concept of Optimal Prediction. IEEE Transactions on Automatic Control, 60:1647$1652,2015$.

[27] M. Zorzi and A. Ferrante. On the estimation of structured covariance matrices. Automatica, 48(9):2145-2151, 2012. 